\title{
PARTIÇÃO DA BIOMASSA E RELAÇÕES BIOMÉTRICAS DE AROEIRA-PIMENTEIRA
}

\author{
BIOMASS PARTITIONING AND BIOMETRICS OF THE BRAZILIAN PEPPERTREE \\ Carlos Roberto Sanquetta ${ }^{1}$, Sérgio Costa Junior ${ }^{2}$, Mateus Niroh Inoue Sanquetta ${ }^{3}$, \\ Ana Paula Dalla Corte ${ }^{4}$, José Henrique Pedrosa Macedo \\ 1, 2, 3, 4, 5 Universidade Federal do Paraná, Curitiba, Paraná, Brasil - carlossanquetta@gmail.com, \\ o.sergio.costa@gmail.com, mateus.sanquetta@gmail.com, anapaulacorte@gmail.com \& \\ johpema2@gmail.com
}

\begin{abstract}
RESUMO
Schinus terebinthifolius Raddi é uma importante espécie nativa de ocorrência no bioma Mata Atlântica. A determinação da biomassa e sua alocação auxiliam na compreensão da distribuição dos multiprodutos de uma floresta. Este trabalho teve como objetivo analisar a distribuição dos diferentes compartimentos da biomassa total em indivíduos de $S$. terebinthifolius com 12 anos no município de Pinhais, estado do Paraná. Trinta árvores foram abatidas e separadas em: fuste, galhos e folhas, e as raízes foram escavadas até $1 \mathrm{~m}$. Amostras correspondentes a cada compartimento foram retiradas e analisadas em laboratório a fim de se obter a relação de teor de umidade. Os dados foram analisados por meio da construção de uma matriz de correlação simples entre a biomassa dos compartimentos e total com variáveis biométricas. Além da biomassa, diâmetro a 1,30 $\mathrm{m}$ do solo e altura total foram mensurados. Os seus valores observados variaram de 5,00 a 11,60 cm, com média de 8,02 cm, e de 6,00 a 8,80 m, com média de 7,52 m, respectivamente. A biomassa seca apresentou variação entre 7,08 a 37,95 kg, com média de $19,05 \mathrm{~kg}$. A distribuição da biomassa foi: fuste, com 51,89\%, galhos com 22,24\%, raízes com $18,33 \%$ e folhas com $7,55 \%$. O fator de expansão de biomassa variou de 1,14 a 2,94, com média de 1,65. A razão de raízes variou de 0,08 a 0,27 , com média de 0,17 . A biomassa se correlacionou significativamente com o diâmetro a 1,30 $\mathrm{m}$ do solo, mas não com a altura e entre si. O FEB apresentou correlação apenas com altura comercial (inversamente) e $R$ com nenhuma das variáveis.
\end{abstract}

PALAVRAS-CHAVE: Alometria, Fator de expansão, Peso seco, Razão de raízes.

\begin{abstract}
Schinus terebinthifolius Raddi is an important native species which occurs in the Atlantic Forest. Biomass determination and its allocation helps on the understanding of the distribution of the multiproducts of a forest. The aim of this study was to analyze the distribution of the different compartments of the total biomass in individuals of S. terebinthifolius with 12 years planted on the Pinhais municipality, state of Paraná. Thirty trees were felled and separated in: stem, branches and leaves, and the roots were dug up to 1 meter. Samples corresponding to each compartment were collected and analyzed in laboratory to obtain the humidity content relation. Data were analyzed through a simple correlation matrix between the biomass of the compartments and total with biometric variables. In addition to biomass, diameter at 1.30 meter of soil and total height were measured. Their observed values ranged from 5.00 to $11.60 \mathrm{~cm}$, with an average of $8.02 \mathrm{~cm}$, and from 6.00 to $8.80 \mathrm{~m}$, with a mean of $7.52 \mathrm{~m}$, respectively. The dry biomass varied between 7.08 and $37.95 \mathrm{~kg}$, with an average of $19.05 \mathrm{~kg}$. The biomass distribution was: stem, with $51.89 \%$, branches with $22.24 \%$, roots with $18.33 \%$ and leaves with $7.55 \%$. The biomass expansion factor ranged from 1.14 to 2.94 , with an average of 1.65 . The root ratio ranged from 0.08 to 0.27 , with an average of 0.17 . The biomass correlated significantly with the diameter at $1.30 \mathrm{~m}$ from the soil, but not with height and with each other. FEB showed correlation only with commercial height (inversely) and $\mathrm{R}$ with none of the variables.
\end{abstract}

KEYWORDS: Allometry, Biomass expansion factor, Dry weight, Root-to-shoot ratio. 


\section{INTRODUÇÃO}

A biomassa é vital para todos os ciclos biogeoquímicos da Terra, sendo importante também para a humanidade, como fonte de energia, fibras e alimento. É na biomassa que estão contidos vários compostos e elementos químicos indispensáveis à vida e é formada a matéria orgânica vital para a agricultura. É nas florestas que se encontra grande parte da biomassa na superfície terrestre (CAIRNS et al., 1997; HOUGHTON et al., 2009).

A biomassa é uma variável, entre outras, importante para compreender os índices de produtividade e analisar o crescimento em um determinado sítio (TACKENBERG, 2007), além de permitir a descrição de interações entre a floresta e os nutrientes presentes no solo. Não obstante, subsidia estudos relacionados ao estoque e a dinâmica de carbono nas florestas (SILVEIRA et al., 2008).

Existem vários métodos para quantificar a biomassa florestal (SANQUETTA, 2002), os quais se dividem em diretos e indiretos. Os métodos diretos consistem em determinações, realizando a compartimentação e separação dos diferentes componentes de uma árvore. No entanto, sua aplicação para grandes extensões de área se torna inviável devido ao alto custo de execução. Já os métodos indiretos baseiam-se em estimativas, implicando no emprego de alguma técnica de modelagem ou até mesmo no uso de técnicas de sensoriamento remoto (TACKENBERG, 2007).

Os métodos indiretos podem ser aplicados em duas vertentes distintas, sendo uma para árvores individuais, $\mathrm{e}$ a segunda para o conjunto de árvores contidas em uma determinada área pré-estabelecida. Nesses métodos as entradas dos modelos se distinguem por serem características inerentes às árvores individuais ou o conjunto delas (SALATI, 1994; REZENDE 2000).

Todavia, os métodos indiretos, de alguma forma, requerem dados de medição direta para poderem ser validados e aplicados. Informações primárias sobre estoque de carbono nas florestas e espécies que a compõem são, portanto, indispensáveis.

Entretanto, além da quantificação da biomassa, faz se necessário o estudo sobre a sua partição entre os diferentes componentes de uma árvore, devido a demanda de multiprodutos (COUTINHO et al., 2015).

Diante do exposto, objetivou-se quantificar e analisar a partição da biomassa de aroeira-pimenteira (Schinus terebinthifolius Raddi), uma importante espécie ocorrente na Mata Atlântica, em compartimentos (fuste, galhos, folhas e raízes) e correlacioná-la com variáveis biométricas, como o diâmetro e a altura das plantas.

\section{MATERIAL E MÉTODOS}

Esta pesquisa foi realizada em uma área experimental da Universidade Federal do Paraná (UFPR) - Estação Experimental Canguiri, localizada no município de Pinhais, estado do Paraná, nas coordenadas geográficas de $25^{\circ} 23^{\prime} 30^{\prime \prime} \mathrm{S}$ e $49^{\circ} 07^{\prime} 30^{\prime \prime} \mathrm{W}$, no primeiro planalto do estado, a uma altitude de $900 \mathrm{~m}$. O local está inserido em um clima subtropical úmido mesotérmico (Cfb), característico por verão ameno e inverno vigoroso, conforme a classificação climática de Köppen (RIBEIRO et al., 2008).

S. terebinthifolius (Anacardiaceae) é uma espécie arbórea, nativa do Brasil e presente em grande parte do território nacional (MARTORELLI et al., 2011), popularmente chamada de aroeira-pimenteira. Possui crescimento acelerado e ampla capacidade de dispersão, ocupando facilmente áreas desprovidas de vegetação, podendo até ser recomendada para a recuperação de áreas degradadas (FERRETI et al., 1995; D'ÁVILA et al., 2010). Em pesquisa com indivíduos da espécie, Sanquetta et al. (2015a) descreveram a variação da densidade básica ao longo do fuste de diferentes indivíduos, o valor médio reportado foi de $0,463 \mathrm{~g} \cdot \mathrm{cm}^{-3}$.

Os dados correspondentes à parte aérea dos indivíduos utilizadas nesta pesquisa foram os mesmos dos descritos por Sanquetta et al. (2015b), utilizando a mesma metodologia.

As raízes dos indivíduos foram escavadas até a profundidade máxima de $1 \mathrm{~m}$. Todas as raízes visíveis foram retiradas e impurezas como partículas de solo foram retiradas, para determinar seu peso úmido.

Individualmente foram coletadas amostras de todos compartimentos com aproximadamente $200 \mathrm{~g}$ objetivando-se a determinação do teor de umidade em laboratório. Para tanto, estas foram pesadas e, em seguida, submetidas à secagem em estufa a $70^{\circ} \mathrm{C}$ até o atingimento de peso constante. A relação individual entre a biomassa seca e úmida de cada amostra foram então utilizadas para o cálculo da biomassa aérea seca total de cada árvore (ba).

Foram calculados o Fator de Expansão da Biomassa (FEB) e a Razão de Raízes (R), conforme Sanquetta et al. (2011) e Schikowski et al. (2015).

$$
F E B=\frac{b_{a e r}}{b_{f u s}}
$$

Em que: $b_{\text {aer }}=$ biomassa seca aérea $(\mathrm{kg})=b_{f u s}+b_{\text {gal }}+b_{f o l}$ $(\mathrm{kg}) ; b_{\text {fus }}=$ biomassa seca do fuste $(\mathrm{kg}) ; B_{\text {gal }}=$ biomassa seca dos galhos $(\mathrm{kg})$; e $b_{f o l}=$ biomassa seca das folhas $(\mathrm{kg})$. 


$$
R=\frac{b_{\text {rai }}}{b_{\text {aer }}}
$$

Em que: $B_{\text {rai }}=$ biomassa seca das raízes $(\mathrm{kg})$.

Foram feitas análises estatísticas descritivas das variáveis diâmetro a altura do peito (dap), altura total ( $h t)$ e altura comercial ( $h c)$, bem como para a biomassa seca de todos os compartimentos analisados., além de FEB e $R$. Ademais, foi efetuada a análise de correlação de tais variáveis por meio do Coeficiente de Correlação de Pearson.

\section{RESULTADOS}

\section{Estatísticas descritivas}

O dap das plantas analisadas variou de 5,00 a 11,60 cm, com média de $8,02 \mathrm{~cm}$. Quanto a $h t$, a variação foi de 6,00 a 8,80 m, com média de 7,52 $\mathrm{m}$. As biomassas secas totais variaram de 7,08 a $37,85 \mathrm{~kg}$, com média de $19,05 \mathrm{~kg}$. FEB variou de 1,14 a 2,94, com média de 1,65. $R$ variou de 0,08 a 0,27 , com média de 0,17 (Tabela 1 ).

Tabela 1. Estatísticas descritivas das variáveis biométricas e de biomassa seca.

\begin{tabular}{ccccc}
\hline Variável & Mínimo & Médio & Máximo & $\begin{array}{c}\text { Desvio } \\
\text { padrão }\end{array}$ \\
\hline dap $(\mathrm{cm})$ & 5,00 & 8,02 & 11,60 & 1,75 \\
\hline$h t(\mathrm{~m})$ & 6,00 & 7,52 & 8,80 & 0,65 \\
\hline$h c(\mathrm{~m})$ & 1,35 & 3,48 & 6,40 & 1,31 \\
\hline$b_{\text {tot }}(\mathrm{kg})$ & 7,08 & 19,05 & 37,85 & 9,08 \\
\hline$b_{\text {fus }}(\mathrm{kg})$ & 3,22 & 9,94 & 27,40 & 5,73 \\
\hline$b_{\text {gal }}(\mathrm{kg})$ & 0,96 & 4,25 & 13,30 & 3,08 \\
\hline$b_{\text {fol }}(\mathrm{kg})$ & 0,17 & 1,42 & 5,37 & 1,06 \\
\hline$b_{\text {rai }}(\mathrm{kg})$ & 1,06 & 3,43 & 6,95 & 1,76 \\
\hline$R$ & 0,08 & 0,17 & 0,27 & 0,05 \\
\hline$F E B$ & 1,14 & 1,65 & 2,94 & 0,36 \\
\hline
\end{tabular}

\section{Partição da biomassa}

A partição média da biomassa seca foi de $51,89 \%$ no fuste, $22,24 \%$ nos galhos, $18,33 \%$ nas raízes e de $7,55 \%$ nas folhas (Figura 1). Foram evidenciadas variações expressivas nos percentuais em cada compartimento da biomassa, sendo que no fuste essa variou de 25,13 a $77,66 \%$, nos galhos de 8,28 a $42,41 \%$, nas folhas de 2,26 a $17,28 \%$ e nas raízes de 8,30 a $29,08 \%$, correspondendo a um coeficiente de variação de 22,14; 43,67; 59,83 e 27,33\%, respectivamente.

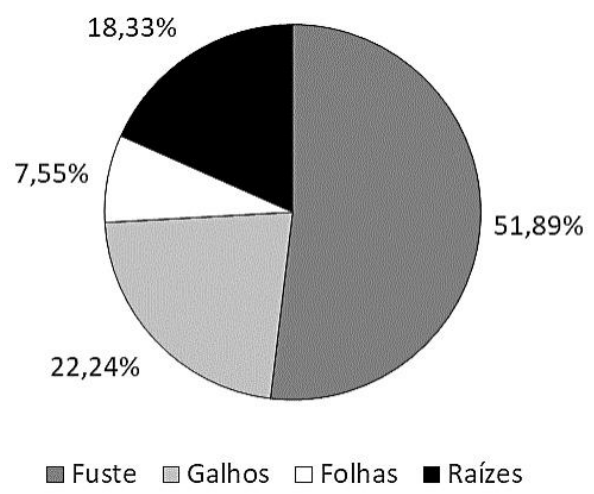

Figura 1. Partição média da biomassa seca.

\section{Correlações}

O dap se correlacionou significativamente com a biomassa total e dos diferentes compartimentos, com coeficientes variando entre 0,62 para biomassa das folhas a 0,89 para biomassa total. Entretanto, dap não se correlacionou com a altura, tanto total $(0,47)$ como comercial $(-0,23)$, tampouco com os fatores de expansão de biomassa $(0,22)$ e razão de raízes $(0,09)$ (Tabela 2$)$. Isso quer dizer que a relação hipsométrica não é forte neste caso. Denota também que altura do ponto de inversão não é afetada pelo diâmetro da árvore, ou seja, ocorrem ramificações mais altas ou mais baixas independentemente se a árvore é mais grossa ou mais fina.

FEB se correlacionou apenas com hc (inversamente) e $R$ com nenhuma das variáveis (Tabela 2 ). Isso indica que árvores com menor altura do fuste (não a total) tendem a apresentar maior fração de biomassa na copa. Isso é coerente porque a existência de ramificações mais próximas da base levará a um maior desenvolvimento da copa e, por conseguinte, maior alocação de biomassa nesse compartimento em detrimento do fuste. Por outro lado, a fração da biomassa referente às raízes não é afetada em si pela partição da biomassa, embora haja correlação significativa da biomassa de raízes com a de galhos.

\section{DISCUSSÃO}

\section{Estatísticas descritivas}

Os valores de $F E B$ realmente podem apresentar variações inter e intraespecíficas. Ampla variação de $F E B$ também foi constatada em plantações florestais de Populus sp. (SCHIKOWSKI et al., 2015) e Pinus sp. (SANQUETTA et al., 2011) no Brasil e em coníferas na GrãBretanha (LEVY et al., 2010). 
Tabela 2. Correlação entre variáveis biométricas e de biomassa seca

\begin{tabular}{ccccccccccc}
\hline & $d a p$ & $h t$ & $h c$ & $b_{\text {tot }}$ & $b_{\text {fus }}$ & $b_{\text {gal }}$ & $b_{\text {fol }}$ & $b_{\text {rai }}$ & $R$ & FEB \\
\hline dap & 1 & - & - & - & - & - & - & - & - \\
$h t$ & 0,47 & 1 & - & - & - & - & - & - & - \\
$h c$ & $-0,23$ & 0,36 & 1 & - & - & - & - & - & - \\
$b_{\text {tot }}$ & 0,89 & 0,43 & $-0,13$ & 1 & - & - & - & - & - \\
$b_{\text {fus }}$ & 0,68 & 0,49 & 0,18 & 0,87 & 1 & - & - & - & - \\
$b_{\text {gal }}$ & 0,66 & 0,08 & $-0,59$ & 0,65 & 0,24 & 1 & - & - & - \\
$b_{\text {fol }}$ & 0,62 & 0,46 & 0,14 & 0,59 & 0,47 & 0,23 & 1 & - & - \\
$b_{\text {rai }}$ & 0,85 & 0,24 & $-0,33$ & 0,82 & 0,53 & 0,69 & 0,5 & 1 & - \\
$R$ & 0,09 & $-0,35$ & $-0,38$ & $-0,13$ & $-0,36$ & 0,07 & $-0,04$ & 0,41 & - \\
FEB & 0,22 & $-0,15$ & $-0,68$ & 0,08 & $-0,37$ & 0,68 & 0,09 & 0,36 & - & - \\
\hline
\end{tabular}

Os valores de $R$ também apresentam comportamento similar. Para Populus sp. (SCHIKOWSKI et al., 2015) foram reportadas variações de 0,14 a 0,60, para Eucalyptus sp. (DALLA CORTE et al., 2015) de 0,05 a 0,12 e para Pinus sp. (SANQUETTA et al., 2011) de 0,05 a 0,60.

Vários fatores, como a idade e o sítio, podem afetar essas variáveis. Estudos correlatos reportam a tendência de diminuição da proporção de biomassa de raízes, bem como de ramos e folhas, ao longo do gradiente de idade (SANQUETTA et al., 2011; DALLA CORTE et al., 2012; SANQUETTA et al., 2014).

\section{Partição da biomassa}

A alocação da biomassa nos diferentes componentes varia de espécie para espécie e em função do status de competição e do sítio. A aroeira-pimenteira é uma espécie que tende a ramificar-se muito facilmente, ou seja, é tipicamente simpodial. No presente caso o fuste representa mais de $50 \%$ da biomassa total e a parte aérea cerca de $82 \%$, com uma participação importante dos galhos (22\%). Espécies tipicamente simpodiais, como a caixeta (Tabebuia cassinoides), por exemplo, podem ter uma partição mais concentrada nos galhos (MELO et al., 2014). Em acácia-negra (Acacia mearnsii), com características arquitetônicas monopodiais, o compartimento fuste chega a representar mais de $70 \%$ da biomassa total (CALDEIRA et al., 2011).

Com relação às raízes, neste trabalho o percentual superou 18\%, em média. Valores menores são frequentemente reportados em outras espécies florestais cultivadas, como em acácia-negra, com 11\% (CALDEIRA et al., 2011) e 16\% em Eucalyptus saligna, Eucalyptus dunnii e Eucalyptus globulus (SCHUMACHER, 1995). Contudo, valores superiores foram registrados para Eucalyptus pellita (45\%) e Eucalyptus camaldulensis (27\%) (LELES et al., 2001). Portanto, essas diferentes são inerentes a características da espécie, da idade e do sítio, bem como a profundidade da escavação e o diâmetro mínimo considerado.

\section{Correlações}

O coeficiente de correlação observado para dap versus altura, tanto total como comercial, de 0,47 e -0,23, respectivamente, representa uma relação hipsométrica não muito evidente. Denota também que a altura comercial (no ponto de inversão) não é afetada pelo diâmetro da árvore, ou seja, as ramificações ocorrem em diferentes alturas independentemente do diâmetro da árvore. Não obstante pode-se afirmar que a fração da biomassa da copa pode ser maior ou menor independentemente de a árvore ter maior ou menor diâmetro.

Vários estudos reportam a tendência de forte correlação da biomassa total ou do fuste com o dap e em menor grau com a altura (MELO et al., 2014; MOGNON et al., 2014; SANQUETTA et al., 2014). Diversos estudos revelam que a biomassa por compartimento (galhos, folhas, raízes) se correlacionam em menor grau ao dap e a altura (VALERIO, 2009; BARBEIRO et al., 2009; RATUCHNE, 2015), o que também foi observado nos resultados dessa pesquisa.

Para Populus foi reportada uma alta correlação entre dap e $F E B$, mas baixa com $R$ (SCHIKOWSKI et al., 2015). Para Eucalyptus isso não foi verificado (DALLA CORTE et al., 2015). Em um estudo com pinhal bravo (Pinus pinaster) em Portugal, concluiu-se que a altura total é a variável que melhor se correlaciona com o FEB (ENES \& FONSECA, 2013), contudo não evidenciado nesse estudo. Em Araucaria angustifolia foram detectadas correlações não significativas do dap com FEB, mas correlações de grau médio da altura e do dap com $R$ (SANQUETTA et al., 2014). Correlações entre FEB e $R$ parecem ser mais incomuns, 
indicando o baixo grau de associação entre essas grandezas, o que também se verificou neste estudo.

\section{CONCLUSÕES}

A maior fração da biomassa de $S$. terebinthifolius está no fuste, seguida dos galhos, raízes e folhas.

O Fator de Expansão de Biomassa e a Razão de Raízes apresentaram grande amplitude nos indivíduos de $S$. terebinthifolius.

O dap apresentou correlação significativa com a biomassa total e dos diferentes compartimentos analisados.

As alturas, total e comercial, não apresentam correlação significativa com a biomassa total e dos diferentes compartimentos analisados.

A espécie apresenta grande variabilidade arquitetônica, o que repercute em diferentes partições da biomassa entre os indivíduos.

\section{REFERÊNCIAS}

BARBEIRO, L.S.S. et al. Equações para estimativa da biomassa individual de Nectandra grandiflora Ness (canela-amarela). Floresta, v.39, n.4, p.833-843, 2009.

CAIRNS, M.A. et al. Root biomass allocation in the world's upland forests, Oecologia, v.111, p.1-11, 1997.

CALDEIRA, M.V.W. et al. Biomassa de povoamento de Acacia mearnsii De Wild., Rio Grande do Sul, Brasil. Scientia Forestalis, v.39, n.90, p.133-141, 2011.

DALLA CORTE, A.P. et al. Fator de expansão de biomassa e razão de raízes-parte aérea para Pinus spp. plantados no Sul do Brasil. Floresta, v. 42, n.4, p.755-768, 2012.

DALLA CORTE, A.P. et al. Fator de expansão de biomassa, razão de raízes-parte aérea e modelos para carbono para Eucalyptus grandis plantados no sul do Brasil. Enciclopédia Biosfera, v.11, n.21, p.107-119, 2015.

D'AVILA, G. et al. The role of avian frugivores on germination and potential seed dispersal of the Brazilian Pepper Schinus terebinthifolius. Biota Neotropica, v.10, n.3, p.45-51, 2010.

ENES, T.; FONSECA, T. Quantificação da biomassa em pinhal bravo recorrendo a fatores de conversão e expansão (BCEFS). Silva Lusitana, v.21, n.1, p.73-85, 2013.

FERRETTI, A.R. et al. Classificação das espécies arbóreas em grupos ecofisiológicos para revegetação com nativas no estado de São Paulo. Florestar Estatístico, v.3, n.7, p.73-77, 1995.

HOUGHTON, R.A. et al. Importance of biomass in the global carbon cycle. Journal of Geophysical Researc, v.114, G00E03, 2009.
LELES, P.S.S. et al. Crescimento, produção e alocação de matéria seca de Eucalyptus camaldulensis e E. pellita sob diferentes espaçamentos na região do cerrado, MG. Scientia Forestalis, n.50, p.77-87, 2001.

LEVY, P.E. et al. Biomass expansion factors and root: shoot ratios for coniferous tree species in Great Britain. Forestry, v.77, n.5, p.421-430, 2010.

MARTORELLI, S.B.F. et al. Efeito anti-inflamatório e cicatrizante de extrato de hidroalcoólico de Schinus terebinthifolius Raddi (aroeira) a $30 \%$ em ora base - estudo "in vivo". International Journal of Dentistry, v.10, n.2, p.80-90, 2011.

MELO, L.C. et at. Estimativa de biomassa e carbono total para árvores de caixeta no Paraná. Pesquisa Florestal Brasileira, v.34, n.77, p.21-29, 2014.

MOGNON. F. et al. Estimativas de biomassa para plantas de bambu do gênero Guadua. Ceres, v.61, n.6, p.900-906, 2014.

RATUCHNE, L.C. Biomassa e carbono: equações e dinâmica em fragmentos de Floresta Ombrófila Mista no Paraná. 2015. 102p. (Tese de doutorado).

RIBEIRO, L. et al. Zoneamento de riscos de incêndios florestais para a Fazenda Experimental do Canguiri, Pinhais (PR). Floresta, v.38, n.3, p.561- 572, 2008.

SANQUETTA, C.R. Métodos de determinação de biomassa florestal. As florestas e o carbono. 1.ed. Curitiba: UFPR, 2002.

SANQUETTA, C.R. et al. Biomass expansion factor and root-toshoot ratio for Pinus in Brazil. Carbon Balance and Management, v.6, n.6, p.1-8, 2011

SANQUETTA, C.R. et al. Estimativa de carbono individual para Araucaria angustifolia. Pesquisa Agropecuária Tropical, v.44, n.1, p.1-8, 2014.

SANQUETTA, M.N.I. et al. Densidade da madeira de Schinus terebinthifolius Raddi. em povoamento puro no município de Pinhais - PR. Enciclopédia Biosfera, v.11, n.22, p.3604-3612, 2015a.

SANQUETTA, M.N.I. et al. Equações de biomassa para aroeirapimenteira (Schinus terebinthifolius Raddi). Enciclopédia Biosfera, v.11, n.22, p.832-840, 2015b.

SCHIKOWSKI, A.B. et al. Fator de expansão de biomassa e razão raízes - parte aérea para Populus sp. Enciclopédia Biosfera, v.11, n.21, p.107-119, 2015.

SCHUMACHER, M.V. Naehrstoffkreislauf in verschiedenen Bestaeden von Eucalyptus saligna (Smith), Eucalyptus dunnii (Maiden) und Eucalyptus globulus (Labillardière) in Rio Grande do Sul, Brasilien. 1995. 167p. (Tese de doutorado).

SILVEIRA, P. et al. O estado da arte na estimativa de biomassa e carbono em formações florestais. Floresta, n.38, v.1, p.185-206, 2008.

SOMOGYI, Z. et al. Indirect methods of large forest biomass estimation. European Journal of Forest Research, v.126, n.2, 
p.197-207, 2006.

TACKENBERG, O. A new method for non-destructive measurement of biomass, growth rates, vertical biomass distribution and dry matter content based on digital image analysis. Annals of Botany, v.99, n. 4, p.777-783, 2007.

VALERIO, A.F. Quantificação e modelagem da biomassa e carbono em plantações de Pinus elliottii Eng. com diferentes idades. 2009. 123p. (Dissertação de mestrado). 